\title{
Juvenile myoclonic epilepsy among sudanese patients: prevalence and eeg characterizations
}

\begin{abstract}
Introduction: Juvenile myoclonic epilepsy (JME) is well recognised, age-related electroclinical, generalised, epileptic syndrome. JME exceeds the $5 \%$ of all epilepsies and around $20 \%$ of all idiopathic generalised epilepsies. The classical presentations consist of myoclonic jerks (MJs), occur early in the morning and precipitated by sleep deprivation. JME is well documented under diagnosed.
\end{abstract}

Aims: The aims were to identify the prevalence and EEGs characteristics among Sudanese patients with JME.

Methods and Patients: A cross-sectional clinical based study that consists of EEGs recording and history reviewing of the all epileptic patients presented to EEG unit of the National Ribat University, and El magzoub neurosciences centre, from March 2003 to May 2012

Results: Forty-four patients with JME have been diagnosed, with an overall prevalence of $2.13 \%$, based on EEG finding of generalised 3.5-6 Hz single, bifid and polyspikes - slow wave complexes on normal brain background activity. The mean age of JME patients at diagnosis was $19.55 \pm 8.98$ years; the majority of patients $(75 \%)$ were between 10 and 30 years. Myoclonic jerks were detected in $90.9 \%$ of the patients with a mean age of onset $10.48 \pm 4.81$ years. Absence attacks were confirmed in $77.27 \%$ of JME patients, and generalised tonic-clonic seizures in $84.1 \%$ of patients with a mean age of onset $13.92 \pm$ 5.65 years, convulsions were detected in $77.3 \%$ of all patients experienced GTCs.

Conclusion: JME among Sudanese epileptic patients has a low prevalence, thus a high rate of under diagnosis. The most important misdiagnosis pitfalls are the low awareness among doctors, who fail to ask about MJs, underestimation of the MJs by parents, who do not seek advice before the occurrence of fits, and the empirical anti-convulsion drugs that may blackout the MJs. EEGs asymmetries led to missing interpretation and misdiagnosis.
Volume 6 Issue 4 - 2017

\author{
M Salah Elmagzoub,' Sami F Abdalla, ${ }^{2}$ Hoiam \\ A Abzeid ${ }^{3}$ \\ 'Department of physiology, National Ribat University, Sudan \\ ${ }^{2}$ Department of Medicine, Almaarefa Colleges, Saudi Arabia \\ ${ }^{3}$ Department of clinical laboratory sciences -AL-Ghad \\ International colleges for medical sciences, Saudi Arabia
}

Correspondence: Sami Fatehi Abdalla, Department of Medicine, Almaarefa Colleges, Saudi Arabia P.O. Box 711666 Riyadh, Saudi Arabia, Tel 966538199174, Fax 966450390I, Email samibillal@gmail.com

Received: March 15, 2017 | Published: April 10, 2017

Keywords: JME, MJs, EEGs

Abbreviations: AEDs, anti-epileptic drugs; EEGs, encephalography; GTCS, generalised tonic-clonic seizures; ILAE, international league against epilepsy; JME, juvenile myoclonic epilepsy; Mjs, myoclonic jerks; PSW, poly spikes-wave; SW, spikewave

\section{Introduction}

JME is a syndrome that appears around puberty and is characterized by seizures with bilateral, single or repetitive, arrhythmic, irregular myoclonic jerks, predominantly in the arms. Some of the patients may fall due to jerks, but usually not associated with loss of consciousness. Often, there are generalized tonic - clonic seizures (GTCS) and less frequent absences. The seizures usually occur shortly after awakening, and are often precipitated by sleep deprivation. ${ }^{1}$ The prevalence of JME estimated to be $5 \%-10 \%$ of all epilepsies, and $18 \%$ of idiopathic generalized epilepsy ${ }^{2}$ similar to Obeid $(10.7 \%),{ }^{3}$ but many studies revealed lower prevalence, particularly those conducted in specialized clinics as it was 4.1 by Genton et al., ${ }^{4}$ Females have been found to outnumbered males with JME. ${ }^{5}$ Typically the JME appears in the second decade, with a peak between 12 and 18 years. ${ }^{6}$ JME is an electroclinical epileptic syndrome thus its diagnosis should be supported by clinical and electrophysiological findings. generally there are A large number of experts agree on two sets of diagnostic criteria, class I and class II, both agreed on jerks that aggravated by sleep deprivation or /and stress. ${ }^{7}$ The major EEG features in JME are short discharges of generalized spike-wave (SW) or poly spikes- wave (PSW) complexes associated with epileptic myoclonic ${ }^{8}$. During a myoclonic seizure, EEG shows irregular 3 to $4 \mathrm{~Hz}$ polyspikeswaves with front central predominance. The EEGs of Seventy four to eighty-one percent of patients with JME displayed 4 to $6 \mathrm{~Hz}$ bilateral polyspikes and slow wave discharges with frontal predominance over a normal background activity.,

The diagnosis of JME is often missed at the onset of seizures. ${ }^{9}$ In a series studies the mean delay between the onset of symptoms and correct diagnosis was close to 8 years: the only diagnosis proposed by the referring physician was JME in $22(16 \%)$ patients only, ${ }^{4}$ decreased to $3.3 \%$ in Indian study, with mean delay of diagnosis reaches $8.6 \pm 7.0$ years. ${ }^{10}$ To the best of our knowledge, there are no concrete epidemiological studies for JME among Sudanese epileptic patients, despite the common agreement among the Sudanese neurologists and neurophysiologists about the respectful number of patients with JME, they deal routinely within their clinics and EEG centers. Therefore our major aim is to detect the prevalence of JME and correlate the EEGs findings with clinical presentation among Sudanese patients.

\section{Patients and methods}

Based on the most frequent patterns of EEGs and clinical features, 44 patients with JME were assembled from 3523 patients, presented to EEG unit of the National Ribat University, and El magzoub neurosciences centre, from March 2003 to May 2012. Any patient who had diagnosed as JME have been recruited and reviewed personally 
or with informants through a preformed questionnaire and the EEGs have been repeated for all of them. The EEG machine we used to record was the Medtronic machine. The EEGs traces were interpreted by an expert clinical neurophysiologist and neurologist. Objectives of the study have been explained for all patients through a phone call and informed consents have been signed by patients or their legally authorized persons.

\section{Results}

Based on the characteristic EEGs changes in JME (the generalised 3.5 - $6 \mathrm{~Hz}$ single, bifid and polyspike-slow wave complexes), ${ }^{3,8}$ we found that the prevalence of JME among abnormal EEGs was found to be 44 patients $(2.13 \%)$. Associated asymmetries of EEGs localizations and / or patterns have been observed in $45.5 \%$ of JME patients. Gender distribution revealed moderate female predominance (56.81\%), while the male was $43.2 \%$. The mean age of JME patients at diagnosis was $19.55 \pm 8.98$ years; the majority of patients $(75 \%)$ were between 10 and 30 years. Myoclonic jerks were detected in $90.9 \%$ of the patients with a mean age of onset $10.48 \pm 4.81$ years. As far as time is concerned, jerks occurred during early morning and in the day time $(59.1 \%, 50 \%)$. Sleep deprivation appears to be the major trigger for MJs in $61.4 \%$ of the patients, other important triggering factors distributed as follow; emotional stress accounted $50 \%$ of the causes whereas, the early morning awakening was $36.4 \%$. Absence attacks were confirmed in $77.27 \%$ of JME patients, and generalised tonic-clonic seizures in $84.1 \%$ of patients with a mean age of onset $13.92 \pm 5.65$ years, convulsions were detected in $77.3 \%$ of all patients experienced GTCs. Only five doctors (11.4\%), from all doctors who referred their patients for EEG test, included the JME among their differential diagnoses. Thirty-eight patients $(86.4 \%)$ were delivered normally and 6 patients $(13.6 \%)$ by caesarian sections, those who showed the positive history of febrile convulsions were $27.3 \%$. Sleep disturbance was found in $40.9 \%$.Memory capacities declined in $79.5 \%$ and school or work performance deteriorated in $86.4 \%$ of JME patients.

\section{Discussion}

The present JME prevalence result among epilepsy patients in Sudan is $2.13 \%$. A similar estimate was determined by the previous work of Fong group, ${ }^{11}$ but a higher estimate was obtained by Ali ${ }^{12}$. This low prevalence can be explained in the light of the report of Bureau ${ }^{13}$ who found that the patients with JME are easily controlled, thus most of them will not be referred to the neurologic centers, and they will be lost without the precise diagnosis. Inconsistent with the result obtained by Murthy et al., ${ }^{14}$ we found that $77.3 \%$ of patients presented with the classic triad symptoms at the time of the diagnosis; he found it only in $17.5 \%$ of patients. ${ }^{14}$ Ninety present of Sudanese JME had MJs lesser than Ali et al., ${ }^{12}$ observation, ${ }^{12}$ the four patients $(10 \%)$ who denied the occurrence of MJs, were already on AEDs, at the time of referral, that may obscure their jerks, as Panyiotobolous \& Loncman ${ }^{15,16}$ stated in two different studies. ${ }^{15,16}$ Myoclonic jerks in Sudanese patients triggered by sleep deprivation in $61.4 \%$ and emotional stress in 52.3\%, partially accorded with da Silva et al., ${ }^{17}$ results. Photic stimulation and television watching were known stimulators of MJs in $13.5 \%$ of Sudanese patients, consistent with the observation of da Saliva et al., ${ }^{17}$ All our patients denied alcohol consumption, although Janz \& da Silva ${ }^{17}$ included it among the major triggers (Figure 1).

All patients with JME showed the characteristic generalized 3.5 - $6 \mathrm{~Hz}$ single, bifid and polyspike-slow wave complexes, on normal background activity. EEG asymmetries have detected in $45.5 \%$ of patients in present study, higher than Montaleni ${ }^{18}$ (38.1\%) and lower than Letourneau ${ }^{19}(53.8 \%){ }^{18,19}$ The EEG records of $18.8 \%$ of our patients showed focal discharges, accorded with Murthy results. ${ }^{14}$ These EEG asymmetries, particularly the focal discharges are not uncommon in JME patients and many investigators accused as the major pitfall of misdiagnosis and may be the same explanation of low prevalence of JME among Sudanese patients. Only five doctors $(11.4 \%)$ who referred their patients to our centres were labelled their referring report with JME diagnosis, something between Genton \& Vijai $^{9,10}$ results but the mean delay of diagnosis of all patients was 4.35 years, in partial accordance with the both studies. ${ }^{9,10}$ It is apparently that the poor awareness about JME syndrome among doctors had an immense role in JME misdiagnosis, reflected in the form of the low prevalence and long means delay time. This premise is confirmed by the success of increasing Spanish doctors awareness in decreasing the mean delay of JME diagnosis from 10.6 years in $1994{ }^{20}$ to 2.4 years in $2001 .^{21}$ The females represent $56.8 \%$ of Sudanese JME patients, against the declaration of ILAE (1985), ${ }^{22}$ that states equal male to female ratio, but this female predominance has been accorded with most recent observations by Camfield \& Ali et al., 5,12 An $88.6 \%$ Sudanese of patients with JME were diagnosed in their second decade of life, compatibles with the conclusion reached by Ali et al., ${ }^{12}$ and the classic characterization of Janz. ${ }^{23}$ The patients in the current study have wide age range (5- 55 years), it is in line with Panyiotobolous ${ }^{15}$ results $(2-40$ years $),{ }^{15}$ but the minimum and maximum ages were higher in Sudanese patients, embodies more delay of diagnosis in Sudanese patients (Table 1-3).

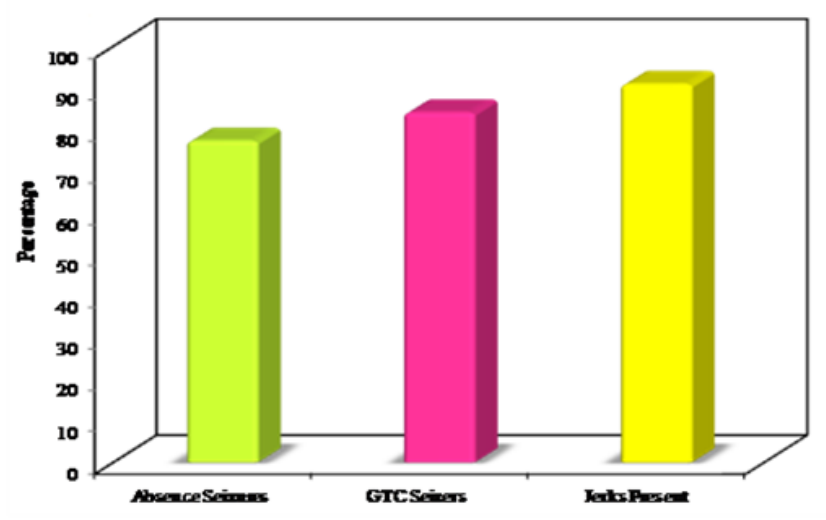

Figure I Frequency of Myoclonic Jerks, GTC and Absences.

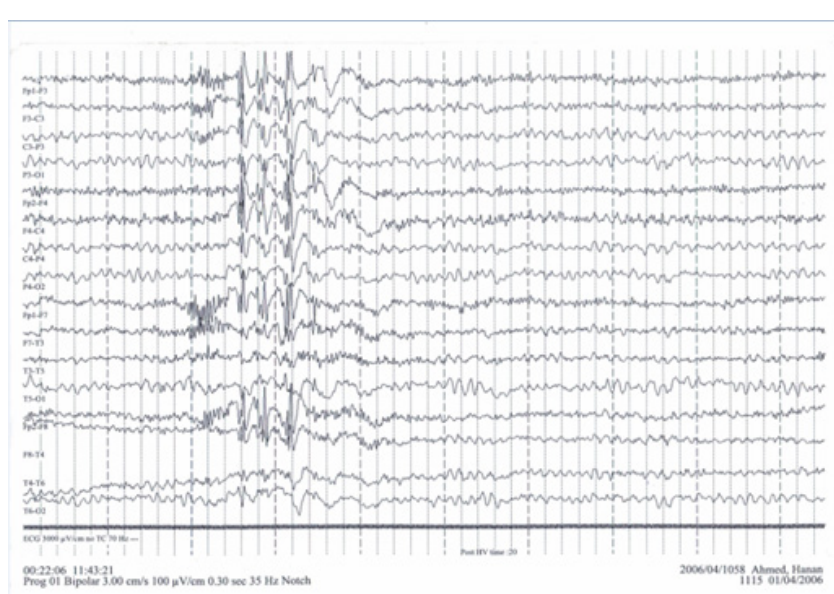

Figure 2 Generalized $3.5-6 \mathrm{~Hz}$ single, bifid and polyspikes - slow wave complexes on normal brain background activity. 
Table I The Prevalence of JME among Patients with Abnormal EEGs

\begin{tabular}{lll}
\hline Abnormal EEGs & No. & $\%$ \\
\hline Juvenile myoclonic epilepsy (JME) & 44 & 2.13 \\
Patients of abnormal EEGs do not include JME patients & 2019 & 97.87 \\
\hline
\end{tabular}

Table 2 The EEGs Findings in patients with JME

\section{Patients Number (2063) EEG Findings}

44

\section{Generalized 3.5-6 Hz single,}

bifid and polyspike - slow wave

complexes on normal cortical background activity

Table 3 Age at Diagnosis of JME

\begin{tabular}{lll}
\hline Age in Years & Number of Patients & Percentage \\
\hline$<10$ & 5 & $\mathrm{II} .4$ \\
$10-<20$ & 22 & 50 \\
$20-<30$ & $\mathrm{II}$ & 25 \\
$\geq 30$ & 6 & 13.6 \\
Min. - Max. & $5.0-55.0$ & \\
Mean \pm SD. & $19.55 \pm 8.98$ & \\
Median & 18 & \\
\hline
\end{tabular}

\section{Conclusion}

It appears that there is no difference between Sudanese patients with JME and the international patients regarding the common EEGs findings.

\section{Acknowledgements}

We acknowledge sister Kadeja Maragan (National Ribat University) and Kholood Sultan(El Magzoub neurological centre) who helped us a lot to complete this work.

\section{Conflicts of interest}

None.

\section{Funding}

None.

\section{References}

1. Fisher RS, van Emde Boas W, Blume W, et al. Epileptic seizures and epilepsy: definitions proposed by the International League Against Epilepsy (ILAE) and the International Bureau for Epilepsy (IBE). Epilepsia. 2014;46 (4):475-482.

2. Appleton R, Beirne M, Acomb B. Photosensitivity in juvenile myoclonic epilepsy. Seizure. 2000; 9(2):108-111.

3. Obeid T, Panayiotopoulos CP. Juvenile myoclonic epilepsy: a study in Saudi Arabia. Epilepsia. 1988;29(3):280-282.

4. Genton P, Gelisse P, Thomas P. Juvenile myoclonic epilepsy today: current definitions and limits. In: Schmitz B and Sander T (Eds.) Juvenile myoclonic epilepsy: the Janz syndrome. Wrightson Biomedical Publishing, Petersfield, England, UK. 2000. p.11-32.

5. Camfield CS, Camfield PR. Juvenile myoclonic epilepsy 25 years after seizure onset: a population-based study. Neurology. 2009;73(13):1041-1045.
6. Delgado Escueta AV, Enrile Bacsal F. Juvenile myoclonic epilepsy of Janz. Neurology. 1984;34(3):285-294.

7. Kasteleijn NolstTrenité DG, Schmitz, B, Janz D, et al. Consensus on diagnosis and management of JME: from founder'sobservations to current trends. Epilepsy Behav. 2013;28(Suppl 1):S87-S90.

8. Hrachovy RA, Frost JD Jr. The EEG in selected generalized seizures. $J$ ClinNeurophysiol. 2006; 23(4):312-332.

9. Genton P, Salas Puig J, Tunon A, et al. Juvenile myoclonic epilepsy and related syndromes: clinical and neurophysiological aspects. In: Malafosse A, Genton P, et al. (Eds.) Idiopathic generalized epilepsies: clinical, experimental and genetic aspects. John Libbey, London, UK. 1994. p.253-265.

10. Vijai J, Cherian PJ, Stlaja PN, et al. Clinical characteristics of a South Indian cohort of juvenile myoclonic epilepsy probands. Seizure. 2003;12(7):490-496.

11. Fong GC, Mak W, Cheng TS, et al. A prevalence study of epilepsy in Hong Kong. Hong Kong Med J. 2003;9(4):252-257.

12. Asadi Pooya AA, Zabihollah Hashemzehi, Mehrdad Emami. Epidemiology and Clinical manifestations of juvenile myoclonic epilepsy (JME) in Iran. Neurol Sci. 2015;38(5):713-716.

13. Bureau M, Genton P, Dravet C, et al. Epileptic syndromes in infancy, childhood and adolescence. 5th edition. Montrouge, France: John Libby Eurotext . 2012. p.305-328.

14. Murthy JM, Rao CM, Meena AK. Clinical observations of juvenile myoclonic epilepsy in 131 patients: a study in South India. Seizure. 1998;7(1):43-47.

15. Panayiotopoulos CP, Obeid T, Waheed G. Absences in juvenile myoclonic epilepsy: a clinical and video-electroencephalographic study. Ann Neurol.1989;25(4):391-397.

16. Loncman ME, Asconape JJ, Penry JK. Clinical and EEG asymmetries in juvenile myoclonic epilepsy. Epilepsia. 1994;35(2):302-306.

17. da Silva Sousa P, Lin K, Garzon E, et al. Self-perception of factors that precipitate or inhibit seizures in juvenile myoclonic epilepsy. Seizure. 2005;14(5):340-346

18. Montalenti E, Imperiale D, Rovera A, et al. Clinical features, EEG findings and diagnostic pitfalls in juvenile myoclonic epilepsy: a series of 63 patients. J Neurol Sci. 2001;184(1):65-70.

19. Létourneau K, Cieuta Walti C, Deacon C. Epileptiformasymetries and treatment response in juvenile myoclonic epilepsy. Can J Neurol Sci. 2010;37(6):826-830

20. Salas Puig J, Tunon A, Vidal JA, et al. Juvenile myoclonic epilepsy of Janz: a frequent and unknown syndrome: 85 patients. Med Clin. 1994;103:684-689.

21. Calleja S, Salas Puig J, Ribacoba R, et al. Evolution of juvenile myoclonic epilepsy treated from the outset with sodium valproate. Seizure. 2001;10(6):424-427.

22. Commission for Classification and Terminology of the International League Against Epilepsy Proposal for classification of epilepsies and epileptic syndromes. Epilepsia. 1985;26(3):268-278.

23. Janz D. Juvenile myoclonic epilepsy. Cleve Clin J Med. 1989;56(Suppl):S23-S33. 